\title{
A TDOA-Based Three-Dimensional Positioning Method for loT
}

\author{
Yu Zhang a, Kai Gao and Jiang Zhu \\ School of Electronic Science, National University of Defense Technology, Changsha 410073, \\ China;
}

azhangpiepie94@163.com

Keywords: 3D positioning, TDOA, Chan algorithm, IoT.

\begin{abstract}
As the Internet of Things (IoT) expands, new networks are initiated in order to provide long-range links with low power and low cost. At the same time, end-devices, localization is attracting more attention. This paper proposes a three-dimensional (3D) positioning algorithm based on time difference of arrival (TDOA). We use unmanned aerial vehicle (UAVs) as gateways to receive signals and calculate, while usual methods are required to construct base stations (BSs). Using UAVs is more flexible and convenient than constructing base stations. Simulation results demonstrate that the proposed method performs better. The second simulation shows that with the increase of the number of BSs, the positioning accuracy improves.
\end{abstract}

\section{Introduction}

With the development of IoT, low-power wide-area network (LPWAN) attracts more attention, which provides remote network access for IoT applications and maintains a low power consumption [1]. LoRa is one of the LPWAN technologies, and it proposes to use three or more gateways to calculate the time difference of arrival (TDOA) of the received signal [2][3]. The power and cost of modules can be minimized by deploying internal positioning technology in the network rather than using GPS-based technology.

Two-dimensional (2D) TDOA location algorithms usually can be divided into two groups: analytic solution and recursive solution. The recursive solution needs an initial condition to improve the positioning accuracy [4], and the initial condition is generally difficult to obtain. So analytic solutions are widely used [5][6]. However, the undefined z-axis error of hyperbola equations of 2D TDOA always causes inaccurate results. In recent years, some TDOA-based 3D positioning methods have been studied [7][8]. However, these methods are available only when there are four and more BSs nearby and when all BSs have the same height [9].

To eliminate the undefined axis error, we propose a new more applicable TDOA algorithm and derivate a hyperbola equation in 3D coordinates that can include z-axis. In the new algorithm, BSs could have different height. We try to replace fixed base stations with UAVs to collecting the information from end-devices and calculating positions. The UAVs install the GPS module so that their position information can be gained. By using UAVS, the network doesn,t need to construct base stations, so it,s convenient and flexible in harsh environments.

This paper is structured as follows. In Section, we introduce the system model for the TDOA based $3 \mathrm{D}$ positioning. In Section, the solution of hyperbola equation based on the $3 \mathrm{D}$ model is given. We present simulation results in Section and conclude the paper in section. 


\section{System Model}

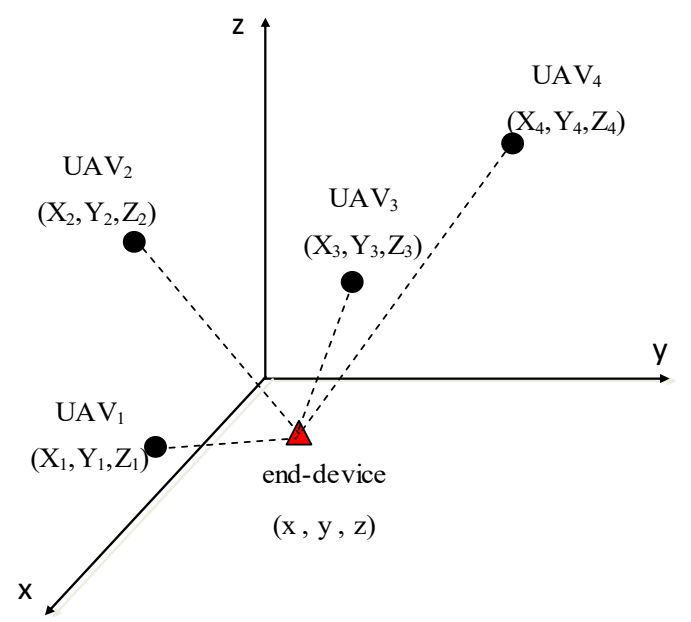

Fig.1 3D Positioning Model

Fig. 1 shows the 3D TDOA model. In this model, UAVs are drove to act as BS to receive the signals. The UAVs install the GPS module so that their position information can be gained. UAVs based IoT is convenient and flexible to calculate positions of end-devices.

Assume that there are M UAVs distributed in a 3-D plane as shown in Fig.1. Let the target be at an unknown position $(x, y, z)$ and the ith UAV at a known location $\left(X_{i}, Y_{i}, Z_{i}\right)$. The squared distance between the target and the ith UAV is

$$
R_{i}^{2}=K_{i}-2 X_{i} x-2 Y_{i} y-2 Z_{i} z+x^{2}+y^{2}+z^{2}
$$

where $K_{i}=X_{i}^{2}+Y_{i}^{2}+Z_{i}^{2}, i=1,2,3 \ldots . \mathrm{c}$ is the signal propagation speed, then

$$
R_{i, 1}=c t_{i, 1}=R_{i}-R_{1}
$$

define a set of nonlinear equations whose solution gives to $(x, y, z)$.

In the 2D position models, the height of BSs and targets is not considered, so the hyperbola Equ.(2) has some errors. InEqu.(2), time difference $t_{i, 1}$ is in $3 \mathrm{D}$ form but the distance difference $R_{i, 1}$ is in $2 \mathrm{D}$, so that the undefined axis error is caused, and the undefined axis error can lead to performance loss $[10]$.

\section{3D Positioning Method}

Firstly, we transform Equ.(2) into another set of equations.

When ${ }^{i}=1$, we can get

$$
R_{i}^{2}=\left(\mathrm{R}_{i, 1}+\mathrm{R}_{1}\right)^{2}=R_{i, 1}^{2}+2 \mathrm{R}_{i, 1} \mathrm{R}_{1}+R_{1}^{2}
$$

$$
R_{1}^{2}=K_{1}-2 X_{1} x-2 Y_{1} y-2 Z_{1} z+x^{2}+y^{2}+z^{2}
$$

From Equ.(3) and (4), we obtain

$$
R_{\mathrm{i}, 1}^{2}+2 R_{i, 1} R_{1}=-2 X_{i, 1} X-2 Y_{i, 1} y-2 Z_{i, 1} Z+K_{i}-K_{1}
$$

where $X_{i, 1}=x_{i}-x_{1}, Y_{i, 1}=y_{i}-y_{1}$ and $Z_{i, 1}=z_{i}-z_{1}$. Note that Equ.(5) is a set of linear equation with unknowns $x, y, z$ and $R_{1}$.

When $\mathrm{M}$ is greater than four, the system is overdetermined. By introducing an intermediate variable, the nonlinear Equ.(5) can be transformed into a set of equations which are linear in the unknown parameters and the intermediate variable. A least-squares (LS) can give their solution. By exploiting the known relation between the intermediate variable and the position coordinates, a second weighted least-squares (WLS) gives the final solution for the position coordinates.

Let $z_{\alpha}=\left[z_{p}^{T}, R_{1}\right]^{T}$ be the unknown vector, where $z_{p}=[x, y, z]^{T}$. We establish a set of linear equations $h=G_{\alpha} z_{\alpha}$ from Equ.(5). With the TDOA noise, the error vector is

$$
\psi=h-G_{\alpha} z_{\alpha}^{0} .
$$


where $h=\frac{1}{2}\left[\begin{array}{c}R_{2,1}^{2}-K_{2}+K_{1} \\ R_{3,1}^{2}-K_{3}+K_{1} \\ \vdots \\ R_{\mathrm{M}, 1}^{2}-K_{M}+K_{1}\end{array}\right], G=-\left[\begin{array}{cccc}X_{2,1} & Y_{2,1} & Z_{2,1} & R_{2,1} \\ X_{3,1} & Y_{3,1} & Z_{3,1} & R_{3,1} \\ \vdots & \vdots & \vdots & \vdots \\ X_{M, 1} & Y_{M, 1} & Z_{M, 1} & R_{M, 1}\end{array}\right]$.

Denote the noise free value of $\{*\}$ as $\{*\}^{0}, t_{i, j}=t_{i, j}{ }^{0}+n_{i, j}, R_{i, 1}=R_{i, 1}{ }^{0}+c n_{i, 1}$. Noting from Equ.(2) that $R_{i}^{0}=R_{i, 1}{ }^{0}+R_{1}^{0}$, the error vector $\psi$ is found to be

$$
\psi=c B n+0.5 c^{2} n \odot n \approx c B n
$$

where $B=\operatorname{diag}\left\{R_{2}^{0}, R_{3}^{0}, \ldots, R_{M}{ }^{0}\right\}$, the symbol $\odot$ represents the Schur product.

$Q$ is the covariance of TDOA estimators, and the covariance matrix of error vector $\psi$ is

$$
\Psi=\mathrm{E}\left[\psi \psi^{T}\right]=c^{2} B \mathrm{E}\left[\mathrm{nn}^{T}\right] B=c^{2} B Q B .
$$

Considering the elements of $z_{\alpha}$ are independent, Equ.(6) can be solved by WLS and the estimate of $z_{\alpha}$ is

$$
z_{\alpha}=\arg \min \left\{\left(\mathrm{h}-\mathrm{G}_{\alpha} z_{\alpha}\right)^{T} \Psi^{-1}\left(\mathrm{~h}-\mathrm{G}_{\alpha} z_{\alpha}\right)\right\}=\left(\mathrm{G}_{\alpha}^{T} \Psi^{-1} \mathrm{G}_{\alpha}\right)^{-1}\left(\mathrm{G}_{\alpha}^{T} \Psi^{-1} \mathrm{~h}\right) .
$$

In practice, $\Psi$ is not known because $B$ contains the true distances between the target and UAVs. To make the problem solvable, replace $\Psi$ with $Q$, the approximation of Equ.(9) is

$$
z_{\alpha} \approx\left(\mathrm{G}_{\alpha}^{T} \mathrm{Q}^{-1} \mathrm{G}_{\alpha}\right)^{-1}\left(\mathrm{G}_{\alpha}^{T} \mathrm{Q}^{-1} \mathrm{~h}\right) .
$$

We firstly obtain an initial estimation of $B$ using Equ.(10). The answer of first step is then computed from Equ.(9). Let $z_{\alpha}=z_{\alpha}{ }^{0}+\Delta z_{\alpha}$, then $\Delta z_{\alpha}$ and its covariance matrix is

$$
\begin{aligned}
& \Delta z_{\alpha}=c\left(\mathrm{G}_{\alpha}^{0 T} \Psi^{-1} \mathrm{G}_{\alpha}^{0}\right)^{-1} \mathrm{G}_{\alpha}^{0 T} \Psi^{-1} B n \\
& \operatorname{cov}\left(\mathrm{z}_{\alpha}\right)=\mathrm{E}\left[\Delta \mathrm{z}_{\alpha} \Delta \mathrm{z}_{\alpha}^{T}\right]=\left(\mathrm{G}_{\alpha}^{0 T} \Psi^{-1} \mathrm{G}_{\alpha}^{0}\right)^{-1} .
\end{aligned}
$$

The solution of $z_{\alpha}$ assumes that $x, y, z, R_{1}$ are independent. But they are related by Equ.(1) at $i=1$. We can use this relationship to give an improved estimate. $z_{\alpha}$ is a random vector with its mean centered at the true value and covariance matrix given by Equ.(11). The elements of $z_{\alpha}$ can be expressed as $z_{\alpha, 1}=x^{0}+e_{1}, z_{\alpha, 2}=y^{0}+e_{2}, z_{\alpha, 3}=z^{0}+e_{3}, z_{\alpha, 4}=R_{1}^{0}+e_{4}$, where $e_{1}, e_{2}, e_{3}$ and $e_{4}$ are estimate errors of $z_{\alpha}$. A new error vector is

$$
\text { where } h^{\prime}=\left[\begin{array}{c}
\left(\mathrm{z}_{\alpha, 1}-\mathrm{X}_{1}\right)^{2} \\
\left(\mathrm{z}_{\alpha, 2}-Y_{1}\right)^{2} \\
\left(\mathrm{z}_{\alpha, 3}-Z_{1}\right)^{2} \\
\mathrm{z}_{\alpha, 4}{ }^{2}
\end{array}\right], G_{\alpha}^{\prime}=\left[\begin{array}{lll}
1 & 0 & 0 \\
0 & 1 & 0 \\
0 & 0 & 1 \\
1 & 1 & 1
\end{array}\right], z_{\alpha}^{\prime}=\left[\begin{array}{c}
\left(\mathrm{x}-\mathrm{X}_{1}\right)^{2} \\
\left(\mathrm{y}-\mathrm{Y}_{1}\right)^{2} \\
\left(\mathrm{z}-\mathrm{Z}_{1}\right)^{2}
\end{array}\right] \text {. }
$$

As the errors $e_{i}, i=1,2,3,4$ are small, the covariance matrix of $\psi^{\prime}$ is

$$
\begin{aligned}
& \Psi^{\prime}=E\left[\psi^{\prime} \psi^{\mathrm{T}}\right]=4 B^{\prime} \operatorname{cov}\left(\mathrm{z}_{\alpha}\right) B^{\prime} \\
& B^{\prime}=\operatorname{diag}\left\{x^{0}-X_{1}, y^{0}-Y_{1}, z^{0}-Z_{1}, R_{1}^{0}\right\}
\end{aligned}
$$

Since is $\Psi^{\prime}$ Gaussian, the ML estimate of $z_{\alpha}{ }^{\prime}$ is

$$
z_{\alpha}^{\prime}=\left(\mathrm{G}_{\alpha}{ }^{\top T} \Psi^{\prime-1} \mathrm{G}_{\alpha}{ }^{\prime}\right)^{-1}\left(\mathrm{G}_{\alpha}{ }^{T} \Psi^{\prime-1} h^{\prime}\right) .
$$

Finally, the estimation of position is then obtained as

$$
z_{p}=\sqrt{z_{\alpha}^{\prime}}+\left[\begin{array}{c}
X_{1} \\
Y_{1} \\
Z_{1}
\end{array}\right] \text {, or } z_{p}=-\sqrt{z_{\alpha}^{\prime}}+\left[\begin{array}{c}
X_{1} \\
Y_{1} \\
Z_{1}
\end{array}\right] .
$$

The proper solution is the one which exists in the region of interest.

\section{Simulation and Analysis}

The root mean square error (RMSE) performance of the proposed method is simulated by Monte Carlo simulations. RMSE is defined as

$$
R M S E=\sqrt{\mathrm{E}\left[(\mathrm{x}-\hat{x})^{2}+(y-\hat{y})^{2}+(z-\hat{z})^{2}\right]}
$$


Figure. 2 shows that the relationship between TDOA measurement error and the RMSE respectively for the proposed method and the traditional method. In the simulation, without loss of generality, we choose these parameters. The target,s position is $(500,1000,10)$. According to the proposed method, some flying UAVs act as base stations, and positions are $(0,0,0),(430$,$2500,200),(720,2500,140),(0,5000,350),(-430,2500,80)$. These known positions are closer to the target,s position and have different height. By contrast, traditional base stations may be further from the target and have lower altitudes. We set these BSi positions are $(0,0,0),(-10000,10000,2),(0$, 10000, 4), (10000, 10000, 12), (10000, -10000, 2).

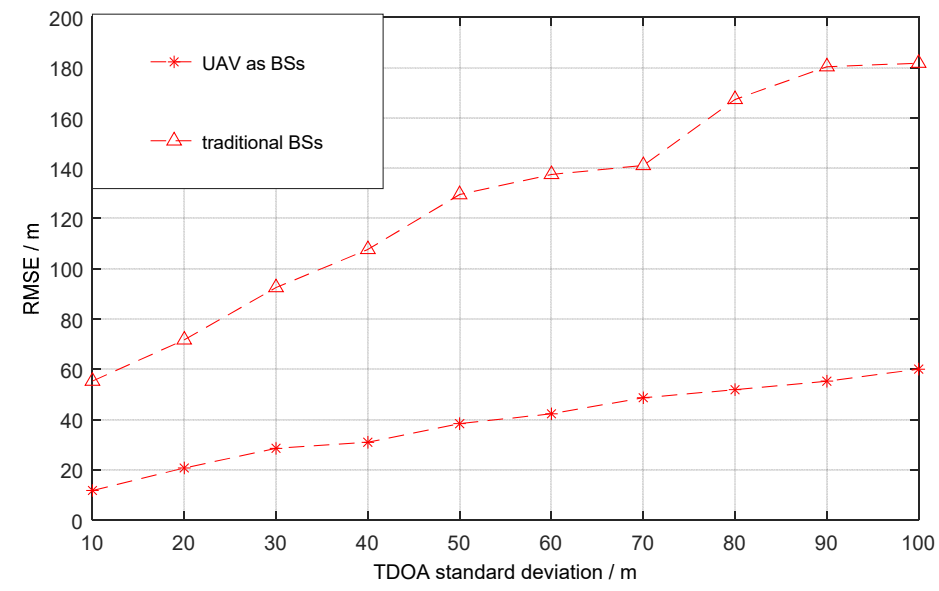

Fig.2Comparisons of the proposed 3D and traditional TDOA algorithm

From the comparisons of 3D and traditional 2DTDOA algorithm shown in Fig.2, the proposed method has a more accurate positioning result. There may be two reasons. First, considering the height of the factors eliminates the coordinate error. Second, UAVs can be closer to the target.

Fig. 3 shows the effect of the UAVs, number on the position accuracy of the proposed method. From [2] we know, when the number of UAVs exceeds 8, the performance doesn,t improve significantly. We discuss the situation where the number is less than 8 . In the simulation, the target, $\mathrm{s}$ position is $(500,1000,10)$, and flying UAVi position is $(0,0,0),(430,-2500,200),(720,2500,140)$, $(0,5000,350),(-430,2500,80),(0,-5000,30),(720,-2500,10)$. From Fig.3, we can conclude that when the number of UAVs is less than 8, as the number increases, the RMSE gets smaller, and the calculated position is closer to the real position.

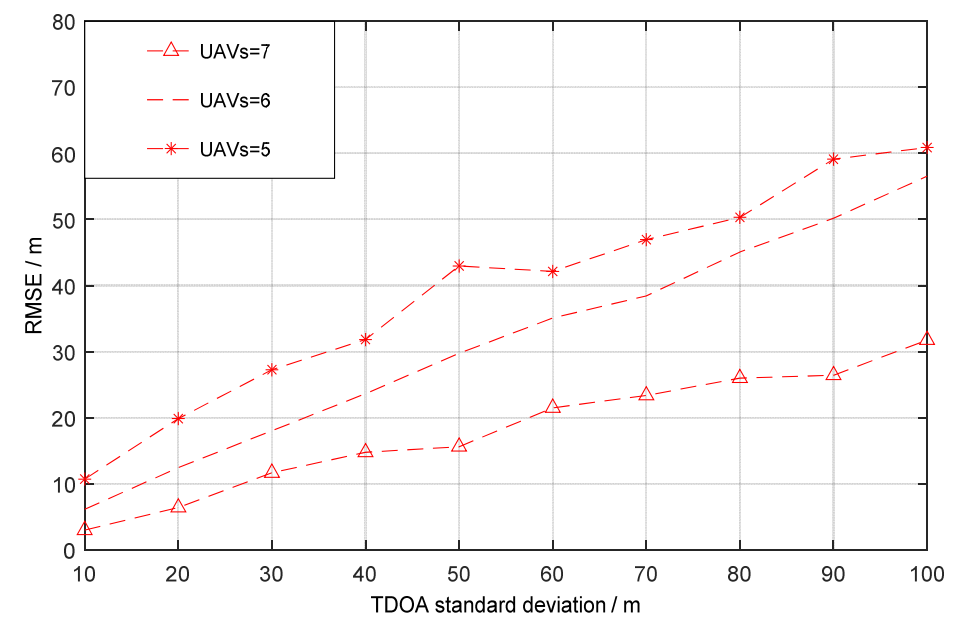

Fig.3The effect of number of UAV

\section{Conclusion}

In this paper, we proposed a $3 \mathrm{D}$ positioning method that can support the implementation in LPWAN. In the 3D model, it is more flexible to find positions using the UAVs as base stations to communicate with end-devices, and it is helpful to improve positioning accuracy. The proposed 
method removes the undefined axis error of 2D TDOA in theory, and it has a better performance than traditional method using fixed base stations which may be far away from end-devices.

\section{References}

[1]. JP Bardyn, T Melly, O Seller. ,IoT: The era of LPWAN is starting now,. European Solid-state Circuits Conference, 2016, p. 25-30.

[2]. Information on: LoRa alliance, https://www.lora-alliance.org/.

[3]. M Bor, J Vidler, U Roedig. ,LoRa for the Internet of Things,. International Conference on Embedded Wireless Systems \& Networks, 2016, p. 361-366.

[4]. W.H. Foy., Position-Location Solutions by Taylor-Series Estimation, IEEE Trans. Aerosp. Electron.Syst., vol. 12 (1976) No. 2, p. 187-194.

[5]. B. T. Fang., Simple Solutions for Hyperbolic and Related Fixes, IEEE Trans. on Aerospace and Electronic Systems, Vol. 26 (1990) No. 5, p. 748-753.

[6]. Chan Y T, Ho K C., A simple and efficient estimator for hyperbolic location, IEEE Trans Signal Proc, Vol. 42 (1994) No. 8, p. 1905-1915.

[7]. H. Zhang et al., An Improved Taylor Series Based Location Algorithm for IEEE 802.15.4a Channels, IEEE Pacific RimConf. Commun., Comput. Signal Process., Aug. 2011, p. 499-503.

[8]. H. Kong, Y. Kwon, and T. Sung., Comparisons of TDOA Triangulation Solutions for Indoor Positioning,. Int. Symp. GNSS,Sydney, Australia, Dec. 6-8, 2004, p. 1-11.

[9]. K Lee, W Hwang. , New TDOA-Based Three-Dimensional Positioning Method for 3GPP LTE System,. Etri Journal, Vol. 39 (2017) No. 2, p. 264-274.

[10]. S. Fischer, Observed Time Difference of Arrival (OTDOA) Positioning in 3GPP LTE, Qualcomm Technologies, Inc. June 2014. 